\title{
Training benefits of virtual bronchoscopy prior to endobronchial ultrasound guide sheath
}

\author{
D. Fielding, F. Bashirzadeh, P. Nguyen
}

Department of Thoracic Medicine, Royal Brisbane and Womens Hospital, Herston, Australia. Email: david_fielding@health.qld.gov.au

Received 4 August 2011; revised 6 September 2011; accepted 16 September 2011.

\begin{abstract}
Research questions: How does a virtual bronchoscopy navigation system (VBNS) improve prediction of candidate bronchus across a range of doctors investigating a range of lesions with endobronchial ultrasound (EBUS) guide sheath? To what extent do benefits of virtual bronchoscopic pre-procedure navigation apply to experienced versus inexperienced bronchoscopists? Methods: Using archived EBUS Guide sheath cases, a comparison was made between identified candidate 4th order bronchus by Computerised tomography (CT) evaluation versus that identified after virtual path creation. Results: From 7 archived cases, 14 doctors identified the correct bronchus in 94 of 98 assessments (95\%). Percentage of cases where there was an improvement in localisation by 2 or more 4th order bronchi was $39.8 \%$ overall $(28.6 \%-51.0 \%), 26.6$ for experienced and 53.1 for inexperienced bronchoscopists $(p<0.02)$. The absolute mean number of 4 th order bronchi different between CT and VBNS was $2.0 \pm 2.6$ overall, 1.2 (range 0 - 6) for experienced, and 2.8 (range 0 - 11) for inexperienced bronchoscopists. Virtual Path software calculation time was $8.1 \pm 2.7$ minutes, compared to $3.6 \pm 2.1$ minutes by CT. Conclusion: VBNS allowed rapid accurate assessment with minimal software training. Greatest benefits in reduction of procedure time were obtained in inexperienced bronchoscopists, and VBNS could allow more rapid skill development in EBUS GS in these doctors.
\end{abstract}

Keywords: Virtual Bronchoscopy; Lung Neoplasms and Solitary Pulmonary Nodule/Diagnosis; Transbronchial Biopsy; Three-Dimensional Imaging; Endobronchial Ultrasonography

\section{INTRODUCTION}

Endobronchial Ultrasound Guide sheath (EBUS GS) sampling of peripheral lung nodules is an accepted method in bronchoscopy [1,2]. Finding small peripheral lesions is difficult [3] and EBUS GS has substantially improved procedural yields [4]. It involves pre procedure scrutiny of the CT chest to predict which bronchus is likely to harbour the lesion into which the probe is passed. However due to a number of factors the selection of the correct bronchus can be difficult. These include the presence of endobronchial variations, difficulty in interpreting segmental pulmonary anatomy on CT, and the experience of the operator $[5,6]$. In recent years a number of virtual navigation tools have improved the ability to extract information from a CT scan which previously would only have been used for axial and coronal imaging $[7,8]$. Now CT reconstruction is used to create "fly through" images showing endobronchial appearances $[9,10]$. Some systems have enough definition to enable correct path tracking in up to 14 branches from the trachea out to a peripheral lesion [11]. The majority identify 5 - 8 branches [9]. This information is particularly useful when combined with EBUS GS because the selection of the bronchus is so critical, thereby reducing procedure time. This has been demonstrated in a limited number of centres, usually by operators highly experienced in the field $[12,13]$. In Asano's series combining EBUS GS with a CT virtual bronchoscopy navigation system (VBNS) the system automatically produced virtual images to a median of fifth- (third- to seventh-) order bronchi [12]. In all patients, the thin bronchoscope could be guided along the planned route, and observation to a median of fifth- (third- to seventh-) order bronchi was possible. Thirty lesions (93.8\%) were successfully visualized by EBUS, and 27 (84.4\%) could be pathologically diagnosed. In lesions $<$ or $=30 \mathrm{~mm}$ in size, the EBUS visualization yield was $91.7 \%$ (22/24), and the diagnostic yield was 79.2\% (19/24). Shinagawa compared use of VBNS with real time in-procedure tracking with EBUS GS to a historical series of VBNS assisted biopsies where the bronchoscopist had to remember the 
images [10]. Here there were slight improvements in diagnostic sensitivity for a similar sized cohort of lesions, as well as a 5 minute reduction in the overall procedure time. This was attributed to the benefits of the VBNS in quickly identifying the correct bronchus. In a recent multicentre study by Asano comparing CT to VB guided EBUS GS there were significant improvements in diagnostic yield ( $81 \%$ for VB versus $67 \%$ for CT) and reductions in examination times [14]. VBNS is not a real time tracking device in itself, that is it does not track the actual bronchoscope; however it has the advantage of simplicity in that it is not directly connected to the patient and does not require additional bronchoscopic hardware. It is almost real time in the sense that the proceduralist can easily progress the stored virtual images ahead of the actual bronchoscopic images to allow the correct path to be followed. It utilises existing CT imaging.

Learning endobronchial anatomy is difficult, particularly when combined with the challenge of matching CT segmental anatomy to endobronchial appearances [9]. Sakurada et al have noted great inter-individual differences in subsegmental bronchial angulation, and difficulties due to bronchial movement during respiration [5]. Conversely actually doing the biopsies and manipulation of the EBUS miniprobe in EBUS GS is no different to a standard transbronchial biopsy, and this part of the procedure does not require any real new skills [4]. Therefore for doctors starting out in EBUS GS assistance in obtaining the correct bronchial anatomy would greatly enhance their results, and indeed possibly their overall motivation to learn the technique.

We wanted to determine the practical aspects of a virtual bronchoscopic navigation system- how easy was it to use, how much time does it take to learn to use it, and how much longer preparation time is involved. We also wanted to know to what extent it could quickly bring inexperienced bronchoscopists up to the level of their more experienced colleagues in finding the correct bronchus into which they might pass the EBUS GS probe.

\section{MATERIALS AND METHODS}

\subsection{Subjects}

Subjects were bronchoscopists with an interest in EBUS guide sheath TBLB. There were seven experienced and seven inexperienced in EBUS GS. In the experienced group all were at consultant level and the mean number EBUS GS cases was 100, range 10 - 250. Inexperienced bronchoscopists had a year of standard bronchoscopy training during which they had observed EBUS GS procedures and were about to start their own procedural learning of this. All had received endobronchial anatomy tuition as part of their standard bronchoscopy training.

\subsection{Study Design}

A prospective single blind crossover study. Only archived de-identified bronchoscopy data and CT images were used from procedures which were part of a normal patient workup and as such no ethics committee submission was made. A prospective single blind crossover study. Only archived de-identified bronchoscopy data and CT images were used from procedures which were part of a normal patient workup and as such no ethics committee submission was made.

\subsection{Methods}

See Figure 1 for a flow diagram of the study design. There were 7 cases for the doctors to review. Each case had previously had an EBUS GS procedure so the correct bronchus was known from that. The task for each doctor look at the CT scan with the lesion and nominate the correct bronchus they thought would reach that lesion. Then they would use the VBNS software with the same CT images and use that to nominate the correct bronchus. They did not use the name of the bronchus, rather just indicate on a picture of an endobronchial view which bronchus they had chosen. (The endobronchial pictures were prepared for each case from fly through images obtained from the CT of each case. Therefore it was more of a real life situation, rather than just having the doctors mark a single standard anatomical chart.) Each doctor received 45 minutes of tuition in the use of the VBNS system. Each doctor therefore placed 2 points on the same bronchial picture for each case-one for the estimate by $\mathrm{CT}$ viewing, the other from viewing the VBNS fly-through path leading to the lesion. The picture was of all bronchi within the bronchial tree, not just the likely candidate lobe. Doctors were asked to mark the bronchus at the 4th order level. Each doctor therefore marked 7 pictures. These pictures were scored by comparing the two marked bronchi to the actual known correct bronchus - were they the same or different, and if different by how many 4th order bronchi were they incorrect? An integer for each doctor for each of the 7 cases was therefore derived. This was 0 if they were correct and 1, 2 or 3 and so on depending on how many 4th order bronchi they were incorrect by. Within a lobe the number of bronchi different was counted directly back towards the nearest correct bronchus. For example marks on RB1a for CT and RB1b for VBNS would be a score of 1 . Where the identified bronchus was in a different lobe from the correct one then all of the 4 th order bronchi in that lobe were counted in addition to counting the number in the correct lobe. Figure 2 gives an example of creation of a path for 1 lesion. A supplemental video shows the fly through for that case. Figure 3 gives an example of a score sheet for 1 case for 1 doctor. To 


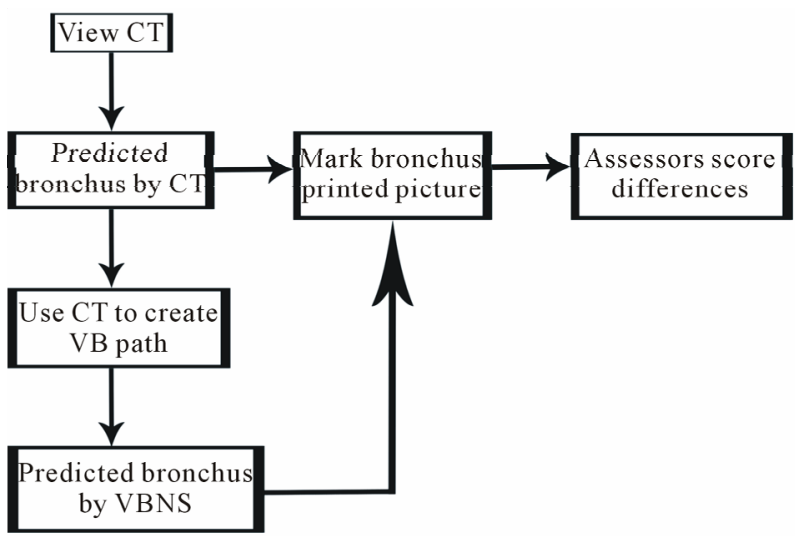

Figure 1. Study design.

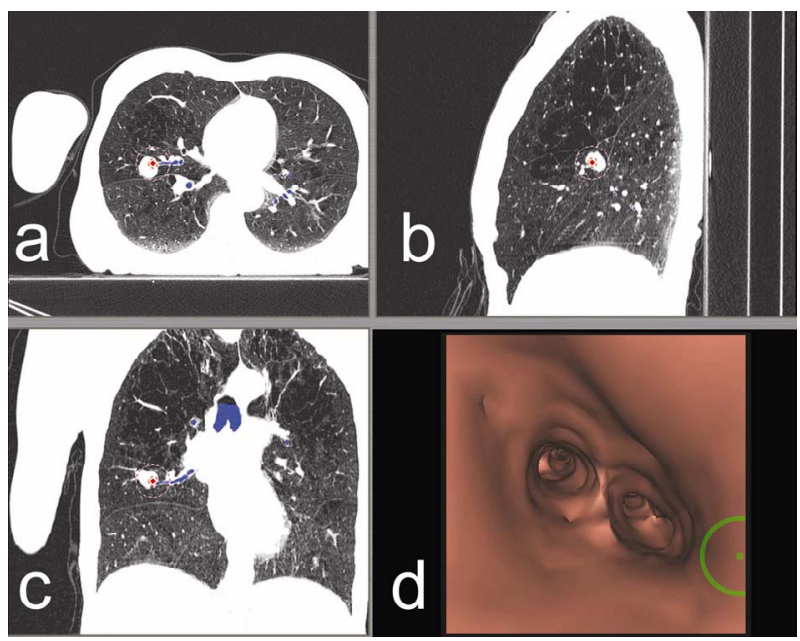

Figure 2. Example of a path created to a lesion in RML using VBNS software. The CT panels ((a) Axial, (b) Coronal, (c) Sagittal) show the blue "bronchogram" which shows a bronchus leading to the lesion. This blue bronchus is clicked by the operator which then leads to the creation of the virtual path down which the operator can scroll ((d) still image from the flythough). The green dot seen in the still example in lower right panel indicated the direction of the lesion.

estimate the added time of missing the correct bronchus by only using CT we timed how long it took to unsuccessfully interrogate a 4th order bronchus in 6 conventional EBUS GS cases, not used in the localisation part of this study.

\subsection{Equipment}

CTs were viewed on Osirix free ware, with both axial and coronal images available. The virtual bronchoscopy system was Olympus VBNS system (Olympus Tokyo 2008). This system inputs Diagnostic Imaging and Commiunication in Medicine (DICOM) CT images of $1 \mathrm{~mm}$ thickness overlapping at $0.5 \mathrm{~mm}$ intervals. The method is as described in Asano, but briefly the operator identifies the lower trachea as the start point, then identifies

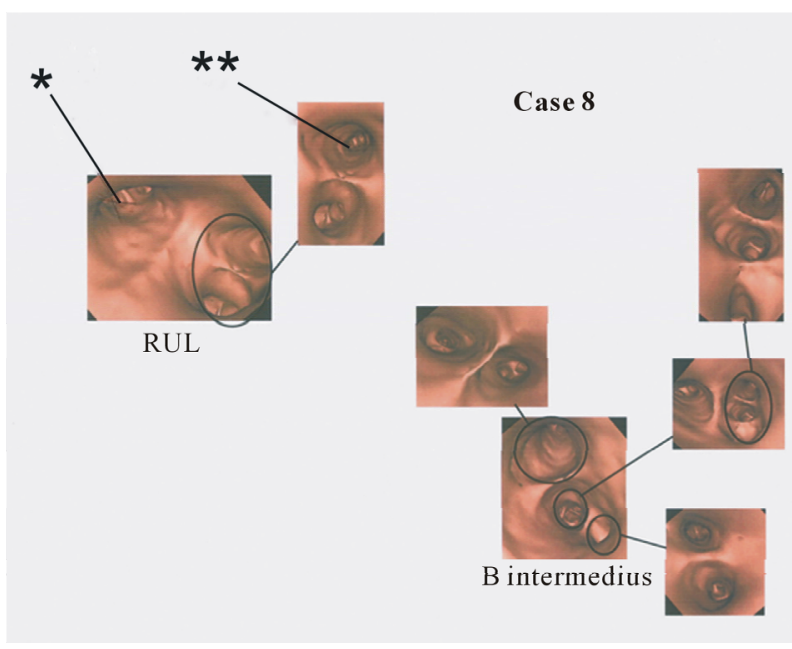

Figure 3. Figure 2 Example of a score sheet of one doctor for one case. The marked bronchus by CT (*) is RB3B. The marked bronchus by VBNS $\left({ }^{* *}\right)$ is RB1b. This is a difference of 4 th order bronchi and a score of 4 is given.

Table 1. Details of archived cases.

\begin{tabular}{cccc}
\hline Case number & Lesion size, mm & Lobe & $4^{\text {th }}$ order bronchus \\
\hline 1 & 70 & RLL & Rb6a \\
2 & 18 & Lingula & LB4a \\
3 & 23 & LUL & LB 1\&2c \\
4 & 11 & LLL & LB 9a \\
5 & 20 & RML & RB4a \\
6 & 10 & RUL & Rb1a \\
7 & 29 & RUL & RB1b \\
Mean & $25.9 \pm 20.6$ & & \\
\hline
\end{tabular}

the pulmonary nodule in question, then identifies the closest bronchus leading to the lesion. The pathway so derived is then presented as a fly through or as a series of thumbnail images at branch points. Archived EBUS GS cases had been performed with Olympus 20 - 20 or 20 - 17 EBUS mini-probes with 4.9 or $6.4 \mathrm{~mm}$ diameter bronchoscopes.

\subsection{Analysis}

Standard statistical comparison of CT versus VBNS such as t tests could not be applied because the data were clumped at zero and skewed above zero.

\section{RESULTS}

Fourteen doctors examined 7 cases, giving a total of 98 sets of data for comparison between CT and VNBS predicted bronchus. Table 2 shows the times taken to review CT data and then create a VBNS path. Overall 
Table 2. Times (in minutes) for review of CT and creation of VBNS path.

\begin{tabular}{cccc}
\hline & All & $\begin{array}{c}\text { Experienced } \\
\text { bronchoscopist }\end{array}$ & $\begin{array}{c}\text { Inexperienced } \\
\text { bronchoscopist }\end{array}$ \\
\hline CT & $3.6 \pm 2.1$ & $4.2 \pm 2.5$ & $2.9 \pm 1.4$ \\
VBNS & $8.1 \pm 2.7$ & $8.8 \pm 2.9$ & $7.4 \pm 2.4$ \\
\hline
\end{tabular}

VBNS added approximately 4 minutes of procedure preparation time. On average experienced bronchoscopists spent a minute longer on reviewing the CT scan, whereas inexperienced bronchoscopists were on average a minute faster in deriving the virtual path candidate bronchus. Overall from the 98, 94 (95\%) correctly identified the candidate bronchus by VBNS. Of the 4 where this was incorrect there was only a single 4th order bronchus different. Figure 1 gives an example of the data sheet for one doctor for one case, with the points for $\mathrm{CT}$ and VBNS identification of the candidate bronchus. Video 1 (supplemental data) is an attachment of a typical virtual bronchoscopic path and bronchus identification. The mean number of 4th order bronchi different between CT and VBNS was as follows: for all cases it was $1.98 \pm$ 2.6, for experienced bronchoscopists it was $1.2 \pm 1.7$, range $0-6$, and for inexperienced bronchoscopists it was $2.8 \pm 3.1$, range $0-11$. In a series of 6 cases where actual EBUS GS was done and a 4th order bronchus was unsuccessfully interrogated we timed that interrogation to take 96 seconds \pm 62 . Using this figure, and applying it to cases where an incorrect candidate bronchus was identified, the approximate maximum time difference at a potential bronchoscopy before the lesion was correctly found would be 9 minutes ( $6 \times 90$ seconds) for experienced bronchoscopists, and potentially 15 minutes $(11 \times$ 90 seconds) for an experienced bronchoscopist. No significant differences in improvement were noted when comparing improvements in localisation of upper lobe lesions versus middle and lower lobe lesions. From the 98 sets of data, the percentage of cases where there was an improvement in localisation by 2 or more 4 th order bronchi was a follows: All cases 39.8, experienced bronchoscopists 26.6 and inexperienced 53.1 ( $\mathrm{p}<0.02$ for experienced versus inexperienced). Using McNemars test, confidence intervals for all cases showed a range of $28.6 \%$ to $51.0 \%$. Therefore it is likely to be a minimum $30 \%$ of procedures in a wider population where some benefit would accrue from using VBNS, in the form of a change of candidate bronchus of 2 or more 4th order bronchi.

\section{DISCUSSION}

The main result of this study was that after a single brief tuition session with the software the correct candidate bronchus could be defined by VBNS in $95 \%$ of cases. This was true for both experienced and inexperienced bronchoscopists and represents quickly acquired accurate information prior to a procedure. In fact inexperienced bronchoscopists on average took a minute less to gain the information, demonstrating quicker uptake of use of the software in this group. The degree of improvement is modest and as expected there was greater benefit for inexperienced bronchoscopists. However in calculating the improvement and a potential time saving we assume that the bronchoscopist would move towards rather than away from the correct bronchus, so potentially the improvements could be an underestimate of benefit. We estimated using the VBNS saves on average around 2 to 3 minutes. This is in keeping with recently published data from a group experienced in VB assisted EBUS GS $[10,18]$. In the ore recent Shinagawa study virtual planning did in fact reduce EBUS GS procedure time by approximately 2 minutes- that study was done by 3 experienced bronchoscopists and our study provides unique information about the potential benefit to trainees as well. As such there would be definite benefits to patient comfort when the predicted bronchus could be improved by up to 6 bronchi in experienced and up to 11 bronchi in inexperienced proceduralists. The strength of our study is the use of actual cases where the gold standard of comparison was the candidate bronchus which had localised the lesion in a real bronchoscopy. Other studies have repeatedly used one endobronchial model simulation for all cases, only varying the target lesion [15]. Merritt et al reported a series of image guided bronchoscopy where lesions had been artificially created on CT. Having created the lesions the 10 bronchoscopists then used the same phantom to reach each of the 10 lesions. Our cases had real lesions which were obviously matched to the real bronchial tree leading to them, and therefore a fully new virtual bronchial path had to be created each time. Nonetheless this group still demonstrated benefits with VB improving overall localisation from $43 \%$ to $94 \%$ for 10 lesions. Interestingly there was no difference between their inexperienced and experienced group in terms of improvement with addition of virtual bronchoscopy. What is the trade off for reducing the procedure time overall by approximately 3 minutes compared to approximately 8 minutes of VBNS preparation beforehand? At least part of the preparation time could be deducted if we subtract the time normally taken for reading the CT, which here was 4 minutes. In addition we believe any time saved with the bronchoscope in situ constitutes a benefit for a patient, although this of course is difficult to quantitate. A procedure shortened by 2 - 3 minutes would likely constitute a comfort benefit to patients, particularly given less manipulation of the 
EBUS GS probe in the bronchus searching for the correct bronchus.

Overall our inexperienced candidates scored very well on their knowledge of CT and endobronchial anatomy before applying VBNS. This may in part reflect their exposure to others performing the procedure and better learning tools now available for training bronchoscopists [16]. In one other series of both experienced and inexperienced bronchoscopists Dolina reported a simulation study where 10 lesions were artificially created at endobronchial sites between 3rd and 5th order bronchi [17]. These lesions were therefore considerably easier to identify and locate than the lesions in this study which were placed at non bronchoscopically visible locations typically at 8th order bronchus level. Similar to our study bronchoscopists recorded their results on a paper decision sheet in this case it was the bronchoscope path to the lesion. Results were that without VB assistance the correct path was only found in $14 \%$, which improved to $49 \%$ with the addition of VB assistance. With a computer tracking tool, perhaps more accurate in terms of the subjects' decisions, there was an improvement from $40 \%$ to $96 \%$ in reaching the target. With either method the basic results for their subjects without the aid of virtual bronchoscopy were therefore lower than our group.

A limitations of our study was that only two of the cases had endobronchial variations, and cases where more variations had been present may well have shown a bigger improvement with VBNS. Also we were not testing manipulation of a bronchoscope to arrive at a certain bronchus as has been done in other mannequin studies. Sakurada et al. [5] noted that some bronchi are more difficult to gain access to such as RB3a and LB 1 \& 2c, however we did not include manual dexterity for access in this study. In terms of the input of CT data into the VBNS system a significant number of cases could not be performed because the CT had not been acquired with thin enough slices, thereby severely reducing definition of virtual images.

In summary we have showed virtual bronchoscopy is an easily aquired skill which improves bronchial localisation, even with a high baseline knowledge of anatomy. Inexperienced bronchoscopists quickly acquire the skill and this affords them greater potential in-procedure benefit. This is in line with the use of virtual tools for a range of bronchoscopic skills [18]. We believe this simple method should be available to all proceduralists doing EBUS GS, but particularly inexperienced bronchoscopists as a compliment to their learning of the EBUS GS method.

\section{ACKNOWLEDGEMENTS}

Supply of VNS software prototype by Olympus Medical Systems
Tokyo.

\section{REFERENCES}

[1] Herth, F.J., Ernst, A. and Becker, H.D. (2002) Endobronchial ultrasound-guided transbronchial lung biopsy in solitary pulmonary nodules and peripheral lesions. European Respiratory Journal, 20, 972-974. doi:10.1183/09031936.02.00032001

[2] Shirakawa, T., Imamura, F., Hamamoto, J., Honda, I., Fukushima, K., Sugimoto, M., et al. (2004) Usefulness of endobronchial ultrasonography for transbronchial lung biopsies of peripheral lung lesions. Respiration, 71, 260-268. doi:10.1159/000077424

[3] Rivera, M.P. and Mehta, A.C. (2007) Initial diagnosis of lung cancer: ACCP evidence-based clinical practice guidelines, 2nd Edition, Chest, 132, S131-148. doi:10.1378/chest.07-1357

[4] Kurimoto, N., Miyazawa, T., Okimasa, S., Maeda, A., Olwa, H., Miyazy, Y., et al. (2004) Endobronchial ultrasonography using a guide sheath increases the ability to diagnose peripheral pulmonary lesions endoscopically. Chest, 126, 959-965. doi:10.1378/chest.126.3.959

[5] Sakurada, A., Takahashi, N., Sato, M., Miyagawa, Y., Matsumura, H. and Murakami, G. (2005) Are difficulties during transbronchial lung biopsy/brush through a fiberoptic bronchoscope based on the bronchial anatomy? Surgical and Radiologic Anatomy, 27, 94-99. doi:10.1007/s00276-004-0297-0

[6] Minami, H., Ando, Y., Nomura, F., Sakai, S. and Shimokata, K. (1994) Interbronchoscopist variability in the diagnosis of lung cancer by flexible bronchoscopy. Chest, 105, 1658-1662. doi:10.1378/chest.105.6.1658

[7] Vining, D.J., Liu, K., Choplin, R.H. and Haponik, E.F. (1996) Virtual bronchoscopy, relationships of virtual reality endobronchial simulations to actual bronchoscopic findings. Chest, 109, 549-553. doi:10.1378/chest.109.2.549

[8] Brillet, P.Y., Fetita, C.I., Biegelman-Aubry, C., Prêteux, F. and Grenier, P.A. (2007) Quantification of bronchial dimensions at MDCT using dedicated software. European Radiology, 17, 1483-1489. doi:10.1007/s00330-006-0496-7

[9] Asano, F. (2010) Virtual bronchoscopic navigation. Clinics in Chest Medicine, 31, 75-85. doi:10.1016/j.ccm.2009.08.007

[10] Shinagawa, N., Yamazaki, K., Onodera, Y., Asahina, H., Kikuchi, E., Asano, F., et al. (2007) Virtual bronchoscopic navigation system shortens the examination time-feasibility study of virtual bronchoscopic navigation system. Lung Cancer, 56, 201-206. doi:10.1016/i.lungcan.2006.12.005

[11] Yu, K.C., Gibbs, J.D., Graham, M.W. and Higgins, W.E. (2010) Image based reporting for bronchoscopy. Journal of Digital Imaging, 23, 39-50. doi:10.1007/s10278-008-9170-8

[12] Asano, F., Matsuno, Y., Tsuzuku, A., Anzai, M., Shinagawa, N., et al. (2008) Diagnosis of peripheral pulmonary lesions using a bronchoscope insertion guidance system combined with endobronchial ultrasonography with a guide sheath. Lung Cancer, 60, 366-373. doi:10.1016/j.lungcan.2007.10.022 
[13] Okisama, S. (2007) Endobronchial ultrasonography with a guide sheath and virtual bronchoscopy navigation aids management of peripheral pulmonary nodules. Hiroshima journal of medical sciences, 56, 19-22.

[14] Asano, F., Yamazaki, K. and Ishida, T. (2008) Usefulness of virtual bronchoscopic navigation in transbronchial biopsy for small pulmonary peripheral lesions: A multicenter, randomized trial. Programs and abstracts of the 15th World Congress for Bronchology, Tokyo, 32.

[15] Merritt, S.A., Gibbs, J.D., Yu, K.C., Patel, V., Rai, L., et al. (2008) Image-guided bronchoscopy for peripheral lung lesions: A phantom study. Chest, 134, 1017-1026. doi:10.1378/chest.08-0603
[16] Colt, H.G., Davoudi, M. and Quadrelli, S. (2007) Pilot study of web-based bronchoscopy education using the essential bronchoscopist in developing countries. Respiration, 74, 358-359.

[17] Dolina, M.Y., Cornish, D.C., Merritt, S.A., Rai, L., Mahraj, R., et al. (2008) Interbronchoscopist variability in endobronchial path selection: A simulation study. Chest, 133, 897-905. doi:10.1378/chest.07-2540

[18] Ishida, T., Asano, F., Yamazaki, K., et al. (2011) Virtual bronchoscopic navigation combined with endobronchial ultrasound to diagnose small peripheral pulmonary lesions: A randomised trial. Thorax. 